\title{
Sensitivity of Occurrence Frequency of Storm Tide Elevation to Random Tidal Phases in Macro-Tidal Area
}

\author{
Siyu (Aaron) Chen, Stantec, Aaron.Chen@stantec.com \\ Mike Anderson, Stantec, Mike.Anderson@stantec.com \\ Jeff Tabar, Stantec, Jeff.Tabar@stantec.com
}

\section{SUMMARY}

Storm tides result from meteorological forces (storm) acting over tidal waters. To derive the reoccurrence frequency of storm tide elevation (STE), of particular interest is to reduce the bias due to random tide selection, since the storm occurrence is independent of tidal phase. This aleatory bias can be reduced by modelling each storm at increasing number of random tides, which, however, adds to the computational cost. Therefore, it is useful to investigate the number of random tides required for each storm to reduce the concerns of aleatory bias. This study firstly explored the nonlinear interaction between tide and surge for a single extratropical (ET) storm in a macrotidal study area. In general, the nonlinearity is 90 degrees out of phase with tide. Although the nonlinearity is important, especially over tidal flats, it is a second order term to the linear combination of tide and surge. This enables the possibility to evaluate the sensitivity of occurrence frequency of storm tides to the number of random tidal phases through a Monte-Carlo Simulation (MCS), where storm tides with surge occurring at random tidal phases can be derived by linear superposition. It was determined that the variance of 100-year STE can be significantly reduced if 10 or more random tides for each storm are considered. It is also possible to eliminate the bias using the MCS with the nonlinearity accounted for by linear regression relating modeled STES and that derived from linear superposition. The accuracy of the approach can be estimated by introducing the regression error randomly to the STEs obtained from linear regression during the analysis.

\section{METHODOLOGY}

Since nonlinearity is expected to be strongest over expansive tidal areas, a single ET storm (ETS) that produced one of the highest water levels over such an area was selected for the investigation. Following Lin et al., 2012, the non-dimensional nonlinearity factor can be defined as

$$
\gamma(t)=\frac{L(t)}{\eta_{\text {storm }}(t)-\eta_{\text {tide }}(t)+H_{\text {tide }}}
$$

with nonlinearity $L(t)=\eta_{\text {storm }}(t)-\eta_{\text {surge }}(t)-\eta_{\text {tide }}(t)$. To drive the nonlinearity, tide-only, surge-only, and eight storm tides (tide + surge) simulations with surge arriving every 1.5 hours to resolve a complete semi-diurnal tide cycle were performed using ADCIRC.

The number of tides $(M)$ that is required to reduce the bias associated with the random tides selection for ET storm can be determined by a MCS procedure as:

1. For a fixed $N, N$ tides are randomly selected for each storm, and storm tides are generated by linear superposition, yielding total $30^{*} N$ storm tides;

2. A return period analysis is performed by fitting the peaks of all storm tides to the generalized extreme distribution (GEV);

3. Steps 1 and 2 are repeated for a sufficiently large number of iterations to yield a consistent mean and standard deviation of the percent-annual-chance STE;
4. Steps 1 through 3 are repeated by incrementing $N$ to yield a unique average and standard deviation of the percent-annual-chance STE. The sufficient number of tides can be determined by identifying the point of diminished returns in terms of reducing the standard deviation with increasing $N$.

\section{RESULTS}

Figure 1 shows the time series $\mathrm{Y}(\mathrm{t})$ for the ETS with surge arriving every 1.5 hours along a tidal cycle with the corresponding tide and surge at a location outside the tidal flats. $Y$ is generally 90 degrees out of phase with the tide during the storm. This is consistent with the study by Lin et al., 2012. The magnitude of nonlinearity is at order of $O(0.1)$, which enables the use of MCS with linear superposition to analyze the variance of occurrence frequency to the number of random tides. Figure 2 shows a plot of the mean and standard deviation of the 100-year STE for $N=1-20$. The inflection point on the standard deviation graph indicates a point of diminishing returns in terms of reducing the variance. By visual inspection, this point occurs between $N=5$ and 10 . Beyond $N=10$, there is little reduction in the standard deviation.

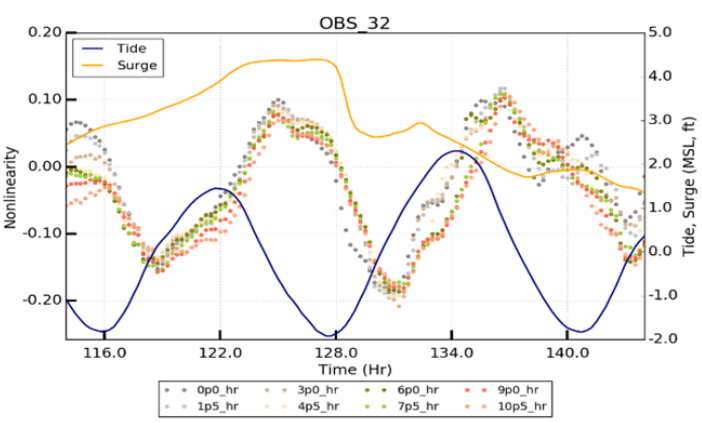

Figure 1: The Nonlinearity Factor for the Eight ETS Simulations at Observation ID 32

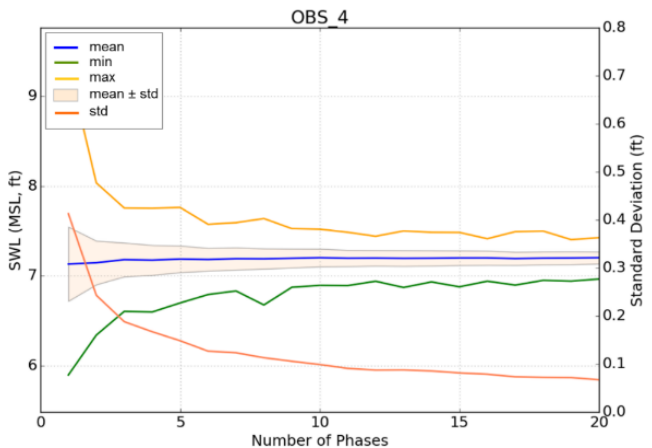

Figure 2: Mean and Standard Deviation of 100 Year STE Using Increasing Number of Tides

\section{REFERENCE}

Lin, Emanuel, Oppenheimer, and Vanmarcke (2012): Physically based assessment of hurricane surge threat under climate change. Nature Climate Change, vol. 10, pp. 462-467. 